\title{
Vielfältige Konzepte der Natur und themenzentrierter Unterricht im Freien anhand der deutschen Übersetzung von Erbens Kytice (Der Blumenstrauß)
}

\section{Zdeněk Vávra}

The aim of the article is to demonstrate the diversity of Nature as it is used within the conceptualization process. The didactic approach of the paper is based on the author's Ph.D. thesis which used the index of pedagogical polarity to support the assumption that teaching with authentic materials is more effective and motivating for both teacher and student. The German edition of Czech writer Karel Jaromír Erben's famous ballad collection Kytice offers diverse inspiration for the activities not only of pupils who speak Czech, but also of those who speak German as their native language. The character of these activities can be cognitive, affective, social and motoric. This topic-centered teaching pursues problem/project and action-oriented goals.

environmental education; nature; literary sciences; foreign language didactics; German language; topic based teaching

Ziel des Artikels ist es zu zeigen, wie vielfältig die Konzeptualisierung der Natur sein kann. Der didaktische Ansatz des Artikels basiert auf der Dissertation des Autors, in der mit Hilfe des Indexes der pädagogischen Polarität verifiziert wurde, dass der Unterricht mit authentischen Materialien im Vergleich zu didaktisierten Materialien effektiver und eher motivierend ist. Die Balladensammlung des tschechischen Schriftstellers Karel Jaromír Erben Der Blumenstrauß in der deutschen Fassung bietet eine vielseitige Inspiration für die Aktivitäten nicht nur von tschechischen, sondern auch von Deutsch als Erstsprache sprechenden Schülern. Die jeweiligen Aktivitäten können kognitiv, affektiv, sozial sowie motorisch ausgerichtet sein. Der themenzentrierte Unterricht verfolgt problem-, projekt- und handlungsorientierte Ziele.

Umwelterziehung; Natur; Literaturwissenschaft; Fremdsprachendidaktik; Deutsch; themenzentrierter Unterricht

\section{Das semantische Feld Natur im Unterrichtsprozess}

Ziel dieses Beitrags ist es nicht, vorrangig literatur- bzw. kulturwissenschaftlich vorzugehen, sondern den Blick eher auf interdisziplinäre Schnittpunkte zu richten, an die im Unterrichtsprozess angeschlossen werden kann. Im ersten Teil des Artikels (Kapitel 2 und 3) werden einige klassische und moderne Konzepte der Natur zusammengefasst; als entscheidendes Kriterium für die Auswahl galt die Möglichkeit, diese Konzepte gedanklich und methodisch im Unterrichtsprozess anhand der Balladen des tschechischen Schriftstellers Karel Jaromír Erben zu entfalten. Im vierten Kapitel wird dem Deutsch sprechenden Leser kurz die Bedeutung von Erbens Kytice (Der Blumenstrauß) für das Nationalbewusstsein im tschechischen Kulturraum und die Übersetzung dieses Klassikers ins Deutsche vorgestellt. Der zweite Teil des Artikels fokussiert auf die Rolle von authentischen Materialien im 
Fremdsprachenunterricht (Kapitel 5), auf die Vorteile und teilweise Risiken des themenzentrierten Unterrichts, insbesondere des themenzentrierten Unterrichts im Freien (Kapitel 6), wobei die Vielfalt der Unterrichtsmöglichkeiten durch konkrete Beispiele veranschaulicht werden soll. Die einschlägigen Unterrichtshilfen sind in Aktivitäten für Deutsch als Erstsprache (Kapitel 7) und Deutsch als Fremdsprache (Kapitel 8) eingeteilt. Einzelne Konzepte der Natur, die Herstellung des Bezugs zum themenzentrierten Unterricht im Freien und der angedeutete didaktische Ansatz fügen sich zu einem Mosaik, das dazu beitragen soll, die deutsche Fassung von Erbens Blumenstrau $\beta$ möglichst anregend und fächerübergreifend in den themenzentrierten Unterricht einzugliedern.

Im Deutschen hat der Ausdruck Natur eine breitere Semantik als das tschechische Pendant príroda. Natur bedeutet auch 'natürlicher Zustand, Charakter, Beschaffenheit'. Daraus ergibt sich, dass die Überlegungen deutscher Philosophen und Pädagogen einen entsprechend größeren Umfang haben. Die Natur kann als das Gesetzmäßige, das Schöpferische oder aber das dem Menschen Gegenüberstehende, im ontologischen Sinne als Wesen des Menschen und im teleologischen Sinne als das zu verfolgende „Werdensziel“ betrachtet werden. Wenn man die Natur als Teil des Menschen auffasst, dann ist zwischen äußerer und innerer Natur zu unterscheiden. In der pädagogischen Theorie steht die Natur einerseits im Gegensatz zur Erziehung, $\mathrm{zu}$ allem Unechten und Künstlichen (z. B. in Rousseaus Prinzip der negativen Erziehung), andererseits ist sie Vorbild für Erziehung und Unterricht. Das Thema Natur kann im Unterrichtsprozess wie folgt behandelt werden: a. kognitiv - als Gegenstand des Erforschens und Kennenlernens; b. affektiv - als Gegenstand des Erlebens; c. sozial - als Ort für gemeinsame Aktivitäten der Schüler; d. motorisch als Gegenstand des Erfahrens (Calließ und Lob 1987: 7).

\section{Klassische Konzepte von Natur}

Die mittelalterliche Philosophie wurde von griechischen Philosophen beeinflusst. Aristoteles (384-322 v. Chr.) klassifizierte als Erster Organismen in hierarchische Stufen: Oben auf der Pyramide platzierte er den Menschen. Alles in der Natur habe seinen Sinn. Pflanzen seien für Tiere und Tiere für Menschen bestimmt (u. a. Nahrung, Kleidung, Instrumente). Im auf Aristoteles basierenden Unterricht tritt die kognitive Fragestellung Wozu? $Z u$ welchem Zweck? auf. Jedoch sieht Aristoteles keinen Grund, sich gegenüber der Natur verantwortungsvoll zu verhalten. Er dachte, dass jeder Verlust von Natur an einem bestimmten Ort zur Bereicherung an einem anderen führe. Die Natur sei aus einem Guss und bilde eine Ganzheit von beständigen Arten (vgl. Kocian 2015: 33).

Im Rahmen der mittelalterlichen Philosophie wurde die Naturvon Gott erschaffen und konnte von Menschen genutzt werden. Der mittelalterliche Mensch lebte in unmittelbarem Kontakt mit der Natur. Tiere betrachtete er als niedriger gestellte 
Wesen, die in einem ähnlichen Verhältnis zum Menschen stehen wie ein Herr und sein Untertan. Die Frage nach der ethischen Verantwortung gegenüber der Natur wurde nicht gestellt. Doch bereits im Mittelalter gab es Denker, die ahnten, dass alle Dinge und Organismen miteinander verbunden sind. Genannt seien Hildegard von Bingen (1098-1179), Franziskus von Assisi (1182-1226) und Gertrud von Helfta (1256-1302) (vgl. Holec et al. 2001: 139). Insbesondere die Gedanken der deutschen Mystikerin, Naturwissenschaftlerin, Heilpraktikerin, Schriftstellerin und Komponistin Hildegard von Bingen sind für die Unterrichtspraxis nach wie vor inspirierend.

Schon Ende des 19. Jahrhunderts wollte man den Naturkundeunterricht anschaulicher gestalten, indem Schulgärten vorgeschlagen wurden. Aus den geplanten Schulgärten entwickelten sich botanische Gärten, wie z. B. der 1929 gegründete Botanische Garten in der bayrischen Stadt Hof, unweit der Tschechischen Republik, belegt. 2001 wurde hier der Heilpflanzengarten und 2004 auch ein Beet mit Pflanzen von Hildegard von Bingen angelegt. Schüler können nach diesem Vorbild selbst kleine Gärten im Stil von Hildegard von Bingen anlegen, die mehrere Funktionen erfüllen:

a) Biologieunterricht (Vermittlung von Kenntnissen über die Heilkraft der Natur);

b) Umweltbildung im Bereich der Erhaltung und Erforschung der Biodiversität;

c) fächerübergreifende Spezialthemen (z. B. Literatur, Geschichte, Fremdsprachen, Dramapädagogik);

d) Entwicklung der motorischen Fähigkeiten (eine anspruchsvollere Variante besteht darin, Freilandflächen der Schulgärten mit Gewächshäusern zu ergänzen);

e) Entwicklung des Verantwortungsbewusstseins;

f) Veranstaltungsort wahrnehmen (Führungen, Verweilen, Meditieren u. ä.) (vgl. Wittig und Niekisch 2014: 462-464).

Johann Amos Comenius (1592-1670) sah die Natur als Vorbild und Ideal. Die Natur sei keine Quelle der Gewalt, sondern eher eine vernünftige Ordnung. Nach Comenius existiert eine Affinität zwischen der äußeren und der inneren Natur des Menschen. Diese Feststellung mündet in den Gedanken, dass die innere Natur des Schülers, sein Sinn für das Gute und für Harmonie mithilfe der äußeren Natur zu entwickeln sei. Die Kenntnis der Natur habe demnach erziehungstechnisch einen praktischen Wert. Comenius leitete einige seiner Grundsätze von Naturgesetzen (die Natur wählt einen passenden Stoff, achtet auf die richtige Zeit, verursacht nichts ohne einen Sinn) ab, von denen an dieser Stelle drei erwähnt werden, die das Konzept des themenzentrierten Unterrichts im Freien theoretisch untermauern:

1. Je mehr Sinne daran beteiligt sind, dass sich eine Vorstellung in die Seele einprägt, desto klarer kann diese Vorstellung verstanden und behalten werden. Als Beispiele nennt er: 
a) eine Erzählung über einen Elefanten hören;

b) einen gemalten Elefanten sehen;

c) dem Elefanten von Angesicht zu Angesicht gegenüberstehen (vgl. Komenský 1946: 52).

Auch in der modernen Zeit empfiehlt u. a. das Bundesministerium für Bildung und Forschung, im Fachunterricht bei der Arbeit mit einem abstrakten Fachtext von einem konkreten Beispiel aus der allgemeinen Alltagserfahrung auszugehen (vgl. Funk und Ohm 1991: 157).

2. Analytische, synthetische und synkritische Methoden sollen unbedingt miteinander verknüpft sein, wenn ein vollständiges Kennenlernen eines Sachverhaltes verlangt wird. Comenius vergleicht diese drei Methoden mit einem Fernglas, einem Vergrößerungsglas und einem Spiegel (vgl. Komenský 1946: 58). Die Möglichkeit, alle drei Methoden im Unterricht im Freien zu verbinden, bieten vor allem handlungsorientiertes Lernen, Projektunterricht und Simulationsspiele mit Pro-und-Contra-Diskussionen.

3. Jeder Schüler sollte sich daran gewöhnen, zugleich auch Lehrer zu sein (vgl. Komenský 1946: 106). Beim Fremdsprachenunterricht wird dadurch einerseits der Wortschatz wiederholt, andererseits dringt man durch das Unterrichten der anderen Schüler tiefer in die Hintergründe ein. Im 20. Jh. hat das Konzept community learning diese Auffassung wiederbelebt: Der Lehrer wird zum Berater und der Schüler zu seinem Klienten. Die Themen werden von den Schülern gewählt, Gruppenarbeit, Partnerschaft und gegenseitige Hilfe überwiegen. Eigeninitiative nimmt einen breiten Raum ein, die Schüler bereiten ihr eigenes Programm mit spontanen Gesprächen, authentischen Texten und Drehbüchern für die Dramatisierung vor (vgl. Choděra und Ries 2000: 71). Die Produkte, Sketche und Spiele werden dann im Wald, auf der Wiese, am Teich, auf einem Hügel, an einer Felswand, in einer Höhle oder - wenn es die Zeit nicht erlaubt - wenigstens auf dem Schulgelände präsentiert.

Die Aufklärung im 17.-18. Jh. ließ eine von den religiösen Vorstellungen befreite Wissenschaft entstehen. Die Natur wurde zum Objekt der wissenschaftlichen Forschung. Erforscht wird das, was messbar oder irgendwie quantifizierbar ist. Die Beherrschung der Natur gehört zum Menschen. In der Vorstellung von Immanuel Kant (1724-1804) werden das Reich der Natur (Notwendigkeit) und das Reich der Freiheit (durch Vernunft begründete Moralgesetze) unterschieden, wobei nur dem Menschen eine Wertschätzung erwiesen werden solle. Kant ist gegen Tierquälerei, weil sie nicht menschenwürdig sei und die Grausamkeit Tieren gegenüber auf Menschen übertragen werden könne (vgl. Kocian 2015: 39). Die Exaktheit und auch eine gewisse methodische Einseitigkeit der Aufklärung ist im heutigen Unterricht immer dann von Vorteil, wenn die Schüler etwas ausprobieren, überprüfen oder auswerten sollen, was vorwiegend bei Experimenten stattfindet. Zu den typischen Merkmalen des Experiments zählen Planmäßigkeit, künstliches Herstellen, 
Wiederholbarkeit und Kontrollierbarkeit. Die Experimente in der Unterrichtspraxis sind allgemeiner aufzufassen als wissenschaftliche Experimente. Hierzu gehören nicht nur Versuche, Messungen und das Experimentieren der Schüler (Einführungs-, Bestätigungs- und Folgeexperiment) sowie praktische Demonstrationen des Lehrers in naturwissenschaftlichen Fächern, sondern auch verschiedene soziale und psychologische Experimente und Selbsterfahrungsexperimente in gesellschaftswissenschaftlichen Fächern und im Fremdsprachenunterricht (Meyer 1987: 313-317).

Eine theoretische Grundlage für Selbsterfahrungsexperimente liefern u. a. Überlegungen von Johann Wolfgang Goethe (1749-1832) und Friedrich Fröbel (1782-1852). Goethe versteht die Natur als eine Dimension, die zum Mittelpunkt der menschlichen Bildung und Selbstverwirklichung gehört. Nach seiner Überzeugung bilden der Mensch und die Natur ein Ganzes. Selbst könne man sich nur entdecken, wenn man auch die Natur kennenlerne. Die Natur ist demnach unser Partner, der uns mit seiner Vielfalt bereichert. Fröbel wurde stärker vom Pantheismus beeinflusst, d. h. von einer Lehre, nach der Gott in allen Dingen der Welt existiert. Die Natur könne zum Spiegel werden, in dem der Mensch sein wirkliches Wesen erkennt. Der Unterricht nach dieser Auffassung animiert Schüler dazu, die Einheit mit der Natur zu erleben, da die Natur kein Gegenstand mehr sei, sondern ein lebendiger Organismus: „Naturgemäße Erziehung wird so zur gesetzmäßigen Erziehung, die zur Einigung mit Gott, mit der Natur, mit der Menschheit, mit dem eigenen Selbst führt" (Calließ und Lob 1987: 10).

Die Autoren der Romantik (1797-1830) zeigen beide Seiten der Natur. Die Gegenwart soll von der Ahnung einer höheren Welt, von einer raum- und zeitlosen Existenz durchdrungen sein. Der Mensch kann in der liebenden Vereinigung mit der Natur die höchste Stufe seiner Selbsterfüllung und Erlösung erleben (u. a. Novalis, Joseph von Eichendorff, Clemens Brentano). Einerseits ist somit die Natur das Reich des Uneingeschränkten, das die Hoffnung auf Entrückung ins Unendliche in sich birgt, andererseits stellten die Romantiker auch ihre Schattenseiten, das Böse und Abgründige dar (u. a. E. T. A. Hoffmann). In der Natur könnten auch dunkle Mächte und dämonische Gewalten walten, statt einem erfüllten Dasein empfinde man hier das Grauen vor der absoluten Leere (vgl. Freund 2000: 98-107). Dieses anscheinend widersprüchliche Denken kann den Unterricht im Freien mit dem Bewusstsein von breiteren Zusammenhängen bereichern. Erst wenn man sich mit lokalen Umweltproblemen bekannt macht, kann man auch globale Zusammenhänge verstehen. Das Vorlesen von Volks- oder Kunstmärchen sowie Balladen bzw. ihre Dramatisierung führt zu dem Wissen, dass im Universum alles enthalten ist, sowohl das Gute als auch das Böse. Trotz der Gefahren, die jeder Aufenthalt im Freien mit sich bringt und die der Lehrer mit den Schülern auch im Voraus bespricht (Vorbeugen ist besser als Heilen: vom Zeckenbiss und Wespenstich über giftige Pflanzen und Pilze bis zu verschiedensten Allergien), sollte man im Unterricht 
vorwiegend das Harmonische eines Aufenthaltes im Freien thematisieren, um eine angemessene Persönlichkeitsentwicklung der Schüler zu fördern und ihren Sinn für das Schöne zu schärfen.

\section{Konzepte von Natur im 20. Jahrhundert}

Der österreichische Esoteriker und Philosoph Rudolf Steiner (1861-1925) war einer der Kritiker des materialistischen Denkens, das sich seit Anfang des 20. Jh. in Europa nach und nach verbreitete. Materialismus setze Grenzen des Wissens, mache die Selbsterfahrung des Menschen und seiner Stellung in der Welt unmöglich. Infolgedessen erziehe der materialistische Lehrer die Schüler zu Neurotikern. Ähnlich wie Goethe betonte auch Steiner, dass man in das Geheimnis und die Schönheit der Natur eintauchen solle. Und ähnlich wie Comenius hob Steiner hervor, dass die Natur eines Menschen freiheitsliebend sei, dass sie weder gehemmt noch gezwungen werden wolle (vgl. Calließ und Lob 1987; Steiner 1993; Komenský 2004). Inspirierend für den heutigen Unterricht ist das Lernen in Etappen, der sog. Blockunterricht, bei dem mehrere Fächer für eine bestimmte Zeit (z. B. zwei Wochen) ein bestimmtes Sachgebiet behandeln. Dieses Sachgebiet kann, je nach den verfügbaren Standortfaktoren, ein Themenkreis (Holz, Waldtiere, Jahreszeiten, Muttertag usw.) oder ein Problemgebiet (Raumplanung, Bebauung von Grünflächen, Abfallwirtschaft, Tourismus usw.) sein. Im Einklang mit Steiner ist es bei Aktivitäten im Freien sinnvoll, den Schülern die Entscheidung zu überlassen, welchen Aspekt des Themas sie behandeln, welche Rolle sie übernehmen und wie sie sich mit dem Problem handlungsorientiert auseinandersetzen. Der Bezug zu den Interessen der Schüler und weitgehende Selbststeuerung fördern die Wahrnehmung von Mitverantwortung. Studien zufolge begünstigt handlungsorientiertes Lernen die multidimensionale Kodierung von Informationen dank der Einbeziehung verschiedener Sinnesorgane und der Verknüpfung der beiden Gehirnhälften (vgl. Gudjons 2001: 84-90). Es entstehen sowohl innere (z. B. Teilnahme an einer Exkursion, Tanz, Singen in einer Fremdsprache, Fest mit ausländischen Mitbürgern, Fantasiereise) als auch äußere Produkte (z. B. Blumenbeet, Fotoausstellung, Schulmarkt, meteorologisches Tagebuch, Figuren aus Kastanien). Die vollkommene Form der Produkte ist dabei nicht so wichtig wie Kreativität, Spontaneität und Spaß am Erleben. Für die Analyse der Erfahrungen und Kenntnisse kann man Formen des Problemunterrichts, heuristische Lösungsverfahren oder auch intuitive Methoden anwenden (vgl. Mazáčová 2008: 50).

Auch die Tänzerin Isadora Duncan (1877-1927) förderte bei ihren Schülern die Entwicklung einer engen Beziehung zur Natur. Sie lehnte die Einschränkungen $a b$, die der klassische Tanz vorschrieb, was sich in ihrer szenischen Präsentation bemerkbar machte (z. B. fliegendes Haar, flatternde Gewänder, darunter ein hautfarbenes Trikot, Barfuß-Tanzen). Der Körper stellte nach ihrer Auffassung 
ein Mittel dar, wie man die Affinität zwischen einer Seele und den Naturgewalten zum Ausdruck bringen kann. Der Unterricht in ihrer Berliner Schule sollte den Kindern eine Naturerfahrung vermitteln. Dazu gehörte Barfuß-Laufen, Wahrnehmen von Bewegungen im Freien (Wolken, Mücken, Blätter usw.) und Erleben der Naturmystik (vgl. Calließ und Lob 1987: 16). Duncan bietet auch heute noch reichlich Inspiration, u. a. für die Integration von Umweltthemen in den Fremdsprachenunterricht in Verbindung mit Bewegungsaktivitäten. Als Beispiel nennen wir die Aktivität Maske zum Thema ,Leben in Afrika'. In der Motivationsphase zeigt der Lehrer Fotos aus Afrika (z. B. Dorfgebiet, Luxusviertel, Armenviertel, Wüste, Savanne, Urwald), die Schüler hören afrikanische Rhythmen, sprechen über Traditionen und Rituale abseits von Reisebüros und Tourismus. $\mathrm{Zu}$ diesen Ritualen gehören auch die Herstellung von Masken und der Tanz mit ihnen. Dieser Tanz drückt Dankbarkeit den guten Kräften der Natur und den Göttern gegenüber sowie Hoffnung auf eine gute Ernte und auf Regen aus. Die bösen Kräfte sollen dadurch verjagt werden. Die Schüler basteln Masken (oval, viereckig oder dreieckig), schmücken sie mit Farben oder Naturstoffen und tanzen mit ihnen im Kreis. Schließlich wird über das Erlebte diskutiert (vgl. Vondrová 2009: 120-121).

Die meisten ethischen Modelle der Natur waren seit der Aufklärung anthropozentrisch. Doch im 20. Jh. erreichen auch andere Richtungen eine neue Blütezeit: Biozentrismus und Theozentrismus. Am Übergang von Anthropozentrismus und Theozentrismus etwa steht der Biozentrismus, der allen Lebewesen einen ethischen Eigenwert zuschreibt. Als Begründer des Biozentrismus gilt Albert Schweitzer (1875-1965). Die Existenz aller Lebewesen ist ähnlich, sie haben auch ihre Ängste, leiden wie die Menschen, sind ebenfalls zum Tod verurteilt. Die Umweltethik von Schweitzer schätzt insbesondere menschliche Werte wie Mitgefühl, Empathie und Rücksicht hoch (Kocian 2015: 57). Der Theozentrismus hält die Natur für eine Gabe Gottes und deshalb ist der Mensch für die Erhaltung der Natur Gott oder einer anderen transzendenten Instanz gegenüber verantwortlich.

Nach der jahrhundertelangen Trennung von Wissenschaft und Religion verlangen etliche Theologen ihre Wiedervereinigung. Genannt seien hier der katholische Theologe Eugen Drewermann (1940) und der protestantische Theologe Jürgen Moltmann (1926). Die Rückkehr zum mittelalterlichen Vermächtnis und zu Denkern wie dem heiligen Franziskus von Assisi, dem alle Geschöpfe (nicht nur die Tiere und Pflanzen, sondern auch das Wasser, die Erde, die Sonne und der Mond) Brüder und Schwestern waren, veranschaulichen die drei von Karl Heinz Peschke (1932) formulierten Prinzipien: 1. Liebe zur Natur; 2. Wertschätzung der Natur; 3. Selbstbeschränkung (alles in Maßen) (vgl. Kocian 2015: 100-101). 


\section{Der Blumenstrauß: Deutsche Übersetzung von Erbens Kytice}

Nun kommen wir zu der Frage, wie man die erwähnten, so unterschiedlichen Konzepte von Natur im Unterricht mithilfe eines Lehrwerkes behandeln und die Schüler für Umweltthemen und zwischenmenschliche Beziehungen sensibilisieren kann. Der Autor dieses Beitrags nutzte im Unterricht als Ausgangspunkt von Überlegungen zu verschiedenen Gestalten der Natur einen Klassiker der tschechischen Literatur, und zwar Kytice (deutscher Titel: Der Blumenstrauß). Die Gedichtsammlung heißt eigentlich vollständig Blumenstrauß aus den Nationalsagen, jedoch etablierte sich die kürzere Bezeichnung (mithin i. F. nur: Der Blumenstrauß). Dieses (auch mehrmals verfilmte, dramatisierte und vertonte) Werk wird schon seit dem 19. Jh. bis heute im Unterricht an tschechischen Schulen verwendet und ist wegen seiner Spannung, formalen Vollkommenheit und zeitlosen Thematik bei Schülern beliebt. Aufgrund des melodischen Rhythmus und der leichten Einprägsamkeit haben sich manche Verszeilen im gesprochenen Tschechischen als Redewendungen erhalten, wie "Klika cvakla, dvére letí - táta vcházi do dveř́ („Klink und Angel knarrend schallen - Und der Vater tritt herein") (Erben 2011: 85) oder "Dnes je čtvrtek, zejtra pátek - šiju, šiju si kabátek“ (,Donnerstag ist’s, Freitag morgen - Will ein Röcklein mir besorgen") (Erben 2011: 85). Von einer dauerhaften, wohl sogar zunehmenden Popularität des Werks in Tschechien zeugt u. a. die Tatsache, dass die Reaktionen des Publikums und der Kritiker auf die Vorstellung im Prager Nationaltheater nach der jüngsten Uraufführung am 28. Februar 2019 (Dramatisierung: Martin Kukučka und Lukáš Trpišovský alias SKUTR, Ilona Smejkalová) durchaus positiv waren (vgl. Štástka 2019).

Dagegen ist Der Blumenstrauß im deutschen Sprachraum praktisch unbekannt (abgesehen von Slawisten, die sich professionell mit der tschechischen Literatur befassen). Um das Werk kurz vorzustellen: Der Autor von Der Blumenstrauß, Karel Jaromír Erben (1811-1870), ist tschechischer Schriftsteller, Historiker und Sammler von Volksliedern und Volksmärchen. Der Blumenstrau $\beta$ enthält Gedichte (vorwiegend Balladen), obwohl Erben selbst sie „Sagen“ und „Lieder“ nannte. Zum ersten Mal erschien die Sammlung 1853, die zweite Auflage von 1861 wurde illustriert und um die Ballade Die Lilie (Lilie) ergänzt. Erben ging von der böhmischen, im weiteren Sinne slawischen, Tradition aus. Die Zeit verläuft in den Gedichten zyklisch, der Raum ist symbolisch (z. B. Wald, Hütte, See), die Naturgewalten umgeben den Menschen, er ist ihnen oft machtlos ausgeliefert. Der Held (nicht selten eine Frau) ist kein Herrscher über die Natur, er muss ethische Regeln beachten und sich anpassen, um zu überleben. Dabei kommt die Dualität der Natur (gute und böse Natur) zum Vorschein.

Der Blumenstrauß erschien in einer deutschen Übersetzung in Wien im Jahre 1900. Die Übersetzung lieferten der tschechische Dichter Eduard Albert und die heute fast vergessene tschechische Literatin Marie Kwaysser, zwischen denen es viele 
Gemeinsamkeiten gab. Da sie zur tschechischen Intelligenz gehörten, waren sie zweisprachig, und zwar, wie damals üblich: deutsch-tschechisch. Beide übersetzten nur im Nebenberuf: Albert war Chirurg, Kwaysser Volksschullehrerin. Beide liebten die Poesie der tschechischen Dichter. Beide übersetzten Erbens Blumenstrauß, um dieses Werk der deutschen Öffentlichkeit näher zu bringen. Der Anlass waren Erfolge der tschechischen Kunst im letzten Jahrzehnt des 19. Jh. (z. B. bei der internationalen Musik- und Theaterausstellung in Wien 1892) (Erben/Nachwort von Pavel Kohn 2011: 260). Ein zweites Mal erschien diese deutsche Übersetzung erst im 21. Jh. - mehr als hundert Jahre nach der ersten. Diese Veröffentlichung wurde vom Deutsch-Tschechischen Zukunftsfonds gefördert. Die zweisprachige Ausgabe wurde von der Künstlerin Rut Kohn illustriert und der tschechische Text von ihr handgeschrieben. Pavel Kohn kommentiert dieses Ereignis in seinem Nachwort:

Der tschechisch-deutsche Blumenstrauß wurde wie ein Blumengeschenk zum zweihundertsten Geburtstag von Karel Jaromír Erben ganz leise und unauffällig geboren. In der deutschen Stadt Passau, ein paar Schritte von der österreichischen und ein paar Kilometer von der tschechischen Grenze entfernt. Und weit weg von Wien, wo er vor länger als 110 Jahren zum ersten Mal auf Deutsch erschienen ist. (Erben/Nachwort von Pavel Kohn 2011: 256)

\section{Authentische Materialien im Fremdsprachenunterricht}

Was sind die Gründe für die Arbeit mit dem Blumenstrauß, also mit authentischem literarischen Material im Unterricht? Als Hauptgrund ist die in der Dissertation des Autors des vorliegenden Beitrags bestätigte Hypothese zu nennen, dass der Unterricht mit authentischen Materialien im Vergleich zum Unterricht mit erstellten bzw. didaktisierten Texten im Lernprozess eine höhere Effizienz aufweist. Die Hypothese ließ sich aufgrund des Indexes der pädagogischen Polarität belegen (mehr zur Berechnung: Pelikán 2004: 216-217). Im Gegensatz zu vereinfachten und didaktisierten Texten (Unterrichtseinheit/UE A) führen authentische Materialien (Unterrichtseinheit/UE B) trotz eines höheren Schwierigkeitsgrades zum Nachdenken und zur Suche nach Ursachen, machen Schülern Spaß und erhöhen ihre Motivation. Zudem stellen sie eine reale Sprachform in konkreten Bereichen (z. B. im Umweltbereich und im Bereich der Globalisierung) dar.

Im Zentrum der Beobachtung standen Schüler von sechsten bis neunten Klassen ausgewählter Prager Grundschulen und die gleichaltrigen Teilnehmer von Sprachkursen im Sprachstudio Rolino. Insgesamt wurden im Frühling 2013 77 Schüler in sieben Gruppen in die Forschung einbezogen. Der Index der pädagogischen Polarität UE A (Arbeitsblatt Was fiegt alles auf der Wiese?) betrug im Durchschnitt 0,41, bei UE B (Arbeitsblatt Mai) belief er sich auf -0,22, d. h. die Arbeit mit authentischen Unterrichtsmaterialien war integrativer und kooperativer, 
wobei die Abweichungen bei unterschiedlichen Lehrern relativ gering waren. Ein höherer Wert des Indexes bei UE A drückt aus, dass sich die Lehrer-SchülerKontakte durch eine erhöhte Domination auszeichneten und dass der Lehrer eine höhere Anzahl strikter Anweisungen benötigte, um den Lernprozess zu leiten. UE $\mathrm{B}$ enthielt dagegen mehr neutrale und weniger dominante Kontakte. Die Arbeit verlief selbstständiger, kreativer und handlungsorientierter. Eine höhere Effizienz in UE B wurde u. a. durch die Ergebnisse eines didaktischen Testes bestätigt. Die Schüler erlebten den Erfolg auf zweierlei Weise: Erstens kam die richtige Lösung bei UE B häufiger vor (UE B: 76-86 \%; UE A: 57-71\%), zweitens konnte der Lehrer abschließend hervorheben, dass der authentische Text vorrangig für deutsche Rezipienten bestimmt war und dass tschechische Schüler, ohne es zu ahnen, den Stoff ebenfalls beherrschten, was ihr Vertrauen in ihre Fremdsprachenkennnisse und letztendlich ihr gesundes Selbstvertrauen stärkte (mehr zur Forschung: Vávra 2013: 253).

\section{Themenzentrierter Unterricht im Freien: Herausforderungen und Chancen}

Ausgehend von der Unterrichtspraxis des Autors des Beitrags und seiner Kollegen, Experten aus Tschechien und Deutschland (Pädagogen aus Prag, Pilsen, Rostock und Krumbach), seien die folgenden Vorteile des themenzentrierten Unterrichts genannt:

- Zusammenwirken von Kognitivismus und Interaktionismus,

- Erwerb allgemeinsprachlicher und einzelsprachlicher Kompetenz (in diesem Fall: Deutsch oder Tschechisch),

- Erwerb von Textkompetenz,

- Verbesserung der Sprachrezeption und -produktion (Konzeptualisierung, Formulierung, Artikulation).

Als Vernetzungsarten sind Assoziationen und semantische Verbindungen empfehlenswert. Nach der Wortfeldtheorie (Gliederung des Wortschatzes in Felder, vgl. Trier 1973: 150-151) ergibt sich die Bedeutung eines Wortes aus seinen Beziehungen zu anderen Wörtern; beim Aktivieren eines Grundelementes werden zugleich unterschiedliche Elemente des Feldes mitaktiviert. Entsprechend kann beispielsweise im Falle der Ballade Der Wassermann (Vodnik) das Grundelement eine der Hauptfiguren (Wassermann) oder ein Oberbegriff (Wasser) sein; je nach ihren Deutschkenntnissen suchen die Schüler - im Text, auf Bildern oder direkt in der Natur - zusammenhängende Elemente, wobei sie jeweils den bestimmten Artikel anführen sollen (das Wasser, der See, der Ufersaum, die Wassertiefe, der Fisch, der Krebs usw.) (vgl. Erben 2011: 194-201).

Genannt seien weitere Vorteile des themenzentrierten Unterrichts anhand der Originalversion oder der deutschen Übersetzung von Erbens Blumenstrauß. Es wird 
handlungs- und problemorientiert vorgegangen. Die aufgegriffenen Themen werden situativ und integrativ bearbeitet. Im Unterricht werden auch Querverbindungen zu anderen Fächern hergestellt. Da die Sammlung Ansatzpunkte sowohl für den Sachunterricht (Tschechisch, Deutsch, Geschichte, Naturkunde) als auch für den Kunstunterricht (Schultheater, Zeichnen, Handarbeiten) bietet, kann man Erbens Gedichte mit Erfolg im fächerübergreifenden Unterricht im Freien (Basis: Thema oder Projekt) anwenden. Obwohl sich der themenzentrierte Unterricht und der Projektunterricht methodisch und inhaltlich in mancher Hinsicht überschneiden und ergänzen (Ziele, Vorteile, Hilfsmittel, Auswertung usw.), scheinen die Vorbereitungsund Durchführungsphase des themenzentrierten Unterrichts im Freien den Lehrer vor noch mehr Herausforderungen zu stellen (vor allem im Hinblick auf detailliertes Planen, passende Rollenzuteilung, Leitung und Koordinierung der Schüleraktivitäten sowie auf wetterbedingte Einflüsse). Im Projektunterricht hingegen sind vom Lehrer eher induktive Verfahren und Flexibilität gefordert (vgl. Kratochvílová 2016: 57-58). Der Blumenstrauß, beispielsweise die Ballade Das goldene Spinnrad (Zlatý kolovrat), ist äußerst geeignet sowohl für kreative Formen (Ausdenken verbindender Geschichten zu den Vokabeln, Dramatisierung, Rollenspiele im Freien usw.) als auch für das Learning by Doing (Stricken/Häkeln, Sticken der Muster, Färben der Textilien usw.) im Sinne von John Dewey (vgl. Singule 1991: 25-35).

Eine bunte Palette von Unterrichtsthemen für den Unterricht im Freien bietet ebenfalls die Ballade Der Heilige Abend (Štědrý den) (Erben 2011: 126-131). Bei der Behandlung dieser Ballade kann man sich methodisch auf einen der vier Kernbereiche konzentrieren; diese sind ein allgemeines Thema (Winterwetter, Weihnachtsbrauchtum, Landleben früher und heute usw.), ein konkreter Anlass (Ausflug, Familienfest, Adventzeit in der Stadt usw.), ein landeskundliches Problem (kulturhistorische Kontexte in Deutschland, Österreich, in der Schweiz und in Tschechien) oder ein bestimmtes Erziehungsziel (vgl. Kratochvílová 2016: 44). Wenn man ein bestimmtes Erziehungsziel verfolgt, wird das Bewusstsein der Schüler nicht nur für Einzelheiten, sondern auch für den ethischen Gesamtzusammenhang geweckt.

Eine gründliche Vorbereitung des Lehrers (Materialien, Requisiten, klare Hinweise, Zeitdauer usw.) erfordern in ähnlicher Weise Simulationsspiele im Freien, bei denen die Schüler durch existentielle Fragen und hochkontroverse Themen angesprochen werden. Die Schüler behalten ihre Identität (ihren bürgerlichen Namen), haben eine klare Verantwortung und Machtbefugnisse und bearbeiten wirklichkeitsnahe Dokumente wie Briefe, Landkarten oder Urkunden (mehr zu dieser Thematik u. a. Jones et al. 1984). Viele Ansatzpunkte für eine Simulation bietet u. a. die Ballade Der Schatz (Poklad) (Erben 2011: 38-53). Die Schüler können reale Funktionen übernehmen (z. B. Zeichnen einer Landkarte, Interviewen der Mutter, Planen des Kinderspieles, Berichten über das Ereignis, Urteilen über die Tat, Philosophieren über die Wahrnehmung der Zeit), beliebt 
sind auch Modernisierungsformen und Vergegenwärtigungen wie Pressekonferenz, Kriminachrichten oder Ermittlung eines Detektivbüros. Dank der Lösung der komplexen Probleme entwickelt sich bei den Schülern vernetztes Denken. Sie strukturieren ihr Wissen, entdecken neue Zusammenhänge und erwerben weitere Kompetenzen wie Handlungsfähigkeit, Sozial- und Moralkompetenz, im Freien darüber hinaus die Umweltkompetenz.

\section{Die von der Balladensammlung ausgehenden Aktivitäten in Deutsch als Erstsprache}

Erbens Blumenstrauß bietet eine umfangreiche Inspiration für die Aktivitäten nicht nur tschechischer (Originaltext im Tschechischunterricht, deutsche Übersetzung im Deutschunterricht), sondern auch mit Deutsch als Erstsprache aufwachsender Schüler. Da sich künftige Tschechischlehrer nach wie vor in Seminaren an tschechischen Universitäten mit dem Blumenstraußbefassen, wobei ihnen eine Reihe von entsprechenden methodischen Hinweisen und Handbüchern zur Verfügung steht, wird in diesem Artikel von der Arbeit mit der tschechischen Fassung abgesehen und nur der Übersetzung ins Deutsche Aufmerksamkeit geschenkt. Dank dieser Übersetzung kann man sich mit dem Werk mittlerweile auch an Schulen im deutschen Sprachraum beschäftigen. Geeignete Fächer und Bereiche sind u. a. Literatur- und Religionsunterricht, Dramapädagogik, interkulturelle Erziehung, Umwelt- und Ethikerziehung. Je nach Altersgruppe (Primar-, Sekundarstufe I. oder II.) kann man Themen wie „Wirken der Naturkräfte“, „Zusammenleben der Menschen“, „Mäßigkeit im Leben“ oder Fragen der Verantwortung, Schuld, Strafe und Vergebung behandeln.

Da den Schülern mit Deutsch als Muttersprache manche Themen bekannt sind, kann man sich mit ihnen komparativ auseinandersetzen. Wenn man z. B. die Ballade Lenore des deutschen Dichters Gottfried August Bürger (1747-94) mit Erbens Ballade Das Brauthemd (Svatebni košile) vergleicht, entdeckt man viele Parallelen: Das Mädchen wartet vergebens auf ihren Geliebten, der nicht aus dem Krieg heimkehrt. Dabei hadert sie mit Gott, weil er sie ungerecht behandelt. Der gefallene Geliebte kommt plötzlich als Totenreiter, führt sie mit sich in den Sarg fort (vgl. Freund 2000: 78). Während Lenore ins Totenreich gebracht wird (der Protest des unangepassten Individuums wird bestraft), kann sich Erbens namenlose Heldin durch Gebete retten (sie hat „nur“ eine gruselige Lektion bekommen):

Berathen warst du, Jungfrau, gut,

Daß du dich gabst in Gottes Hut,

Dem schlimmen Feinde zeigtest Muth

(Erben 2011: 79). 
Die Dramatisierung dieses Gedichtes kann man mit einem Ausflug zu einer Burgruine, einem alten Friedhof oder einer Kirche verbinden, die beeindruckende Kulissen für Rollenspiele im Freien bieten können.

Eine typisch slawische Sage erscheint in der Ballade Das Täubchen (Holoubek): Die Seele eines Unschuldigen verwandelt sich in eine weiße Taube (dagegen würde die Seele eines Verbrechers zu einem Raben) (Erben 1988: 155). Auf der Textebene könnte man drei Entschlüsselungsstrategien verwenden, wie die Schüler ihre Leseerwartung formulieren können: 1. Voraussagen über den Inhalt anhand der Überschrift (Das Täubchen); 2. Voraussagen über den Inhalt anhand eines Verses (z. B. „Seht, ein kleiner Eichbaum / Hat sich drauf erhoben / Und ein weißes Täubchen / Sitzt gerne droben") (Erben 2011: 140); 3. Voraussagen über den Inhalt anhand der Illustrationen (vgl. Buhlmann und Fearns 1987: 236).

Die Bilder kann der Lehrer verschiedenen Ausgaben entnehmen, in denen die Ballade von Karel Souček, Miroslav Huptych, František Vrobel, Josef Hlaváček oder Jan Zrzavý illustriert wurde. Empfehlenswert ist die tschechische Ausgabe von 1988 mit Illustrationen des berühmten tschechischen Malers Jan Zrzavý. Der Vorteil seiner Bilder besteht darin, dass sie eine zeitlich und räumlich offene Interpretation (Spekulationen, spontane Aussagen, Fantasiereisen usw.) ermöglichen, was die Kreativität der Schüler fördert. Die Dissertation des Autors des vorliegenden Artikels bestätigte eine höhere Effizienz des Lernprozesses bei der Verwendung dieser Bilder. Angewandt wurde eine verbal-projektive Methode mit einem Test durch unvollständige Sätze (vgl. Vávra 2013: 239-244). Das Thema „Tauben“ kann man fächerübergreifend behandeln (z. B. die Taube als Symbol, Leben der Tauben in der Stadt, ökonomische und gesundheitliche Aspekte der von Tauben angerichteten Schäden, Maßnahmen verschiedener Stadtverwaltungen wie in Zürich, München oder Wien und ihre Wirksamkeit), abschließend verfassen die Schüler Essays zu dieser Problematik, erstellen Poster oder basteln Warnschilder (z. B. Füttern verboten).

In der Europäischen Union sollte sich jedes Land auch mit der Geschichte und Kultur seiner unmittelbaren Nachbarn beschäftigen. Da Erbens Blumenstrauß zu den Hauptwerken der tschechischen Literatur gehört, könnten auch deutsche und österreichische Schüler ihren Blick für tschechische Traditionen schärfen. Erben war zwar Patriot, der vorrangig das Nationalbewusstsein des tschechischen Volkes wachrufen wollte, aber nicht zuletzt sind seine Themen wie Ausdauer, Mut und Schutz contra Schwäche, Angst und Hilflosigkeit überregional und könnten somit ein willkommener Lernstoff sein, der zu interkultureller Kommunikationsfähigkeit und zum Abbau von Vorurteilen über ein anderes Land beiträgt. In diesem Zusammenhang kann man die erwähnte Ballade Das Brauthemd als recht europäisch bezeichnen: Ein ähnliches Motiv ist u. a. in einer serbischen, russischen, polnischen, litauischen, bretonischen und - wie schon erwähnt - in einer deutschen Sage zu finden, d. h. auch unter geographisch und sprachlich entfernten Völkern, was wohl 
auf einen gemeinsamen Ursprung in alten Zeiten zurückzuführen ist (vgl. Erben 1988: 152).

Der Autor des vorliegenden Artikels führte im Juli 2019 eine Umfrage im Landkreis Rostock durch. Befragt wurden Schüler im Durchschnittsalter von 12 Jahren, die die tschechische Balladensammlung nicht kannten. Die Frage lautete: „Welches Thema macht dich neugierig?" bzw. „Von welcher Geschichte möchtest du mehr erfahren?". Die Schüler erhielten farbige Karten mit Namen der Balladen und hatten die Aufgabe, sich für einen Titel zu entscheiden. Drei Balladen erhielten insgesamt mehr als die Hälfte aller Stimmen (der Anteil der Stimmen in eckigen Klammern): 1. Der Fluch der Tochter (Dcerina kletba) [22 \%]; 2. Der Schatz (Poklad) [20\%]; 3. Der Wassermann (Vodnik) [16 \%]. Somit könnte man die Hypothese aufstellen, dass diese Balladen für den Unterricht in deutschsprachigen Ländern besonders gut geeignet sein könnten, da schon allein die Titel das Interesse der Schüler geweckt haben.

Die in Tschechien allgemein bekannte Ballade Die Mittagsdrude (Polednice) hingegen nahm der Umfrage zufolge unter Erbens Balladen die letzte Stelle ein. Obwohl sich die Befragten für diese Ballade nicht entschieden, weckte auch sie ihr Interesse. Häufig wurde die Frage gestellt, was das Wort Drude eigentlich bedeute. Dieses in Norddeutschland kaum gebräuchliche Wort erklärte der Autor der Umfrage den Schülern wie folgt: Es ist eine mal gute, dann wieder böse Hexe, die eher für die Sagen des Alpengebietes, Niederösterreichs und Böhmens charakteristisch ist. Aufgrund der Reaktionen der Schüler lässt sich vermuten, dass auch diese Ballade für den Unterricht geeignet wäre (z. B. Dramatisierung im Freien). Im Vorfeld könnten jüngere Schüler die Bilder von guten und bösen Wesen in verschiedenen Kulturen malen. Ältere Schüler könnten sich Sequenzen aus dem gleichnamigen tschechischen Film Polednice des Regisseurs Jiří Sádek (2016) anschauen, der ein altes Thema (ein Wesen, das von elf bis zwölf Uhr Menschen übel mitspielt) neu bearbeitet hat (eine auf dem Lande unter psychischem Druck lebende Alleinstehende). Ähnlich könnten sich tschechische Schüler im DaF-Unterricht mit dem Thema auseinandersetzen.

\section{Der Blumenstrauß in deutscher Fassung im DaF-Unterricht}

Der Einsatz des Blumenstraußes in deutscher Fassung im DaF-Unterricht bietet viele Sprechanlässe und quasi-natürliche Kommunikationssituationen. $\mathrm{Zu}$ den Funktionen literarischer Texte im Fremdsprachenunterricht zählen u. a. die Effektivierung der Arbeit an fremdsprachigen Kenntnissen und Fertigkeiten, die Erweiterung des Wortschatzes (Systematisierung, Aktivierung und Reflexion), die Festigung grammatischer Kenntnisse (z. B. Diminutiva, Imperativ, Ordinalzahlen, Deklination der Adjektive), der Erwerb pragma-linguistischer Kenntnisse, die Förderung der Persönlichkeitsentwicklung der Lerner, die Dynamisierung und Auflockerung des Unterrichtsgeschehens sowie der Einblick in das kulturelle Leben 
des jeweiligen Landes (Henrici und Riemer 1994: 289). In diesem Fall können sich Lerner in Tschechien auf ihre Kenntnisse der tschechischen Literatur stützen, was die Rezeption erleichtert.

$\mathrm{Zu}$ den Kriterien für die Auswahl der Balladen gehören die Orientierung an den Lernern und ihren Voraussetzungen (Deutschkenntnisse, Alter, Interessen, sprachlich homogene oder heterogene Gruppe, andere Fremdsprachenkenntnisse usw.), die Bestimmung des Schwierigkeitsgrades (einfacher sind kürzere Balladen mit leicht überschaubaren Gesamtstrukturen), die themenzentrierte Lernzielorientierung und die Integrierbarkeit der Texte in Unterrichtseinheiten im Freien. Die Vorentlastung findet üblicherweise im Klassenzimmer statt. Um unbekannte Wörter zu entschlüsseln, hilft der Lehrer den Schülern mit Illustrationen und Gegenständen oder, beim Aufenthalt im Freien, mit Hinweisen auf konkrete Gegebenheiten. $\mathrm{Zu}$ weiteren Entschlüsselungsstrategien gehören auf der Wortebene Umschreibung, Beschreibung, Generalisierung, Exemplifizierung oder Relationen wie Grund/Folge (vgl. Buhlmann und Fearns 1987: 236). Auf der Satzebene sind Illustrationen (z. B. Zuordnung von Illustrationen und passendem Textteil), Signal- und Schlüsselwörter oder Pantomime ebenfalls hilfreich. Auf der Textebene verwenden wir verschiedene Lesestile (vor allem orientierendes und selektives Lesen), Entscheidungs- oder Ergänzungsübungen und autonomes Lernen durch Unterstreichen, Exzerpieren und Vergleichen (die Schüler können sich mit Arbeitsblättern in der Natur frei bewegen, so dass eine angenehme Lernatmosphäre geschaffen wird).

Auch wenn Der Blumenstrauß viele Impulse für das kognitive Lernen liefert (Themen wie Jahreszeiten, Blumen, Bäume), sollte man das affektive, soziale und motorische Lernen im DaF-Unterricht dennoch nicht außer Acht lassen. Das Thema Natur wird affektiv behandelt, indem die Schüler Formen, Klänge und andere Sinneseindrücke wahrnehmen (z. B. am Flussufer oder im Wald), sozial, indem Pantomime, Tanz, spielerische und dramapädagogische Unterrichtshilfen Anwendung finden, und motorisch, indem der Learning-by-Doing-Unterricht möglichst auf die Interessen derjenigen Schüler ausgerichtet ist, die sich den Lernstoff durch unmittelbares Herstellen und Praktizieren aneignen (Sticken von Mustern, Binden von kleinen Sträußen, Herstellung von Kränzen u. a.). Damit der themenzentrierte Unterricht anhand der deutschen Übersetzung von Erbens Blumenstrauß problem-, projekt- und handlungsorientierte Ziele verfolgt, die über die fachimmanenten Zielsetzungen der einzelnen Fächer hinausgehen, sollte nach jeder abgeschlossenen Unterrichtseinheit eine Auswertung erfolgen. Einerseits wird das Lernergebnis vom Lehrer und von den Schülern selbst ausgewertet, andererseits wird „die Qualität des (kooperativen) Arbeitsprozesses als Grundlage guter Ergebnisse“ (Bastian, Combe und Langer 2016: 86) beurteilt. 


\section{Schlussbemerkung}

An dieser Stelle können nicht alle in Frage kommenden Methoden genannt und erklärt werden. Für geeignet hält der Autor des vorliegenden Beitrags Methoden, die eine themenzentrierte Interaktion und eine Individualisierung des Lerntempos bei der Auseinandersetzung mit dem Inhalt der jeweiligen Ballade ermöglichen, wie Stationenlernen, Selbsterfahrungsexperiment, Experten-Methode/Lernen durch Lehren (vgl. Brenner und Brenner 2011: 35-52). Detaillierte Informationen zu Rollenspielen und anderen aktivierenden Methoden in Bezug auf Vorbereitung, Vorentlastung, Material, Zuteilung der Schüler zu Gruppen, ungefähre Dauer oder Didaktik liefern auch tschechische Autoren (vgl. Sitná 2013; Vávra 2013). Weiterführende und offene Fragen wie ausführliche Informationen zum didaktischen und methodischen Kontext sowie evaluierte Erfahrungen und Auswertung der Eignung einzelner Balladen von Erben für den themenzentrierten Unterricht im Freien könnten Gegenstand einer an diesen Artikel anschießenden Studie werden. Der vorliegende Beitrag darf als Ausgangspunkt weiterer didaktischer Ansätze anhand der deutschen Übersetzung von Erbens Kytice betrachtet werden, aus denen ein spannender und effizienter Unterricht resultieren sollte.

\section{Literaturverzeichnis}

Bastian, Johannes / Combe, Arno / Langer, Roman (2016): Feedback-Methoden: Erprobte Konzepte, evaluierte Erfahrungen. Weinheim [u. a.], Beltz.

Brenner, Gerd / Brenner, Kira (2011): Methoden für alle Fächer. Sekundarstufe I und II. Berlin, Cornelsen.

Buhlmann, Rosemarie / Fearns, Anneliese (1987): Handbuch des Fachsprachenunterrichts. Berlin [u. a.], Langenscheidt.

Calließ, Jörg / Lob, Reinhold. (1987): Handbuch Praxis der Umwelt-und Friedenserziehung, Band 2. Umwelterziehung. Düsseldorf, Pädagogischer Verlag Schwann-Bagel.

Choděra, Radomír / Ries, Lumír (2000): Výuka cizich jazyki na prabu nového století (I, II). Ostrava, Ostravská univerzita.

Erben, Karel Jaromír (1988): Kytice. Praha, Odeon.

Erben, Karel Jaromír (2011): Der Blumenstrauß. Kytice. Passau, Karl Stutz.

Freund, Winfried (2000): Deutsche Literatur. Köln, DuMont Buchverlag.

Funk, Hermann / Ohm, Udo (1991): Handreichung Fachsprache in der Berufsausbildung. Zur sprachlichen Förderung von jungen Ausländern und Aussiedlern. Bonn, Bundesminister für Bildung und Wissenschaft.

Gudjons, Herbert (2001): Handlungsorientiert lehren und lernen: Schüleraktivierung Selbständigkeit - Projektarbeit. Bad Heilbrunn/Obb, Verlag Julius Klinkhardt.

Henrici, Gert / Riemer, Claudia (Hgg.) (1996): Einführung in die Didaktik des Unterrichts Deutsch als Fremdsprache mit Videobeispielen. Band 2. Baltmannsweiler, Schneider Hohengehren / Schneider Verlag GmbH.

Holec, Peter / Erdelská, Olga / Černý, Milan / Hrabovec, Ivan / Böhmová, Blanka / Krajčík, Štefan / Hanušovská, Eva / Šrobár, Štefan / Plašienková, Zlatica / Ebringer, Libor / Džatko, Michal / Šimúth, Jozef: (2001): Krestanstvo \& biológia. Trnava, Spolok sv. Vojtecha. 
Jones, Ken / Edelhoff, Christoph / Meinhold, Monika / Oakley, Colin (1984): Simulationen im Fremdsprachenunterricht: Handbuch. München, Max Hueber Verlag.

Kocian, Matúš (2015): Etika životního prostředí. Praha, Lesnická práce.

Komenský, Jan Amos (1946): Didaktika analytická. Praha, Samcovo knihkupectví.

Komenský, Jan Amos (2004): Didaktika analytická. Pưvodni dílo je stylisticky upraveno a doplnèno poznámkami o možnostech praktického uplatnèni některých zásad a pravidel J. A. Komenského na současných základnich školách. Brno, Tvořivá škola.

Kratochvílová, Jana (2016): Teorie a praxe projektové výuky. Brno, Masarykova univerzita.

Mazáčová, Nataša (2008): Vybrané pedagogické inovace v současné škole. Praha, Univerzita Karlova v Praze, Pedagogická fakulta.

Meyer, Hilbert (1987): Unterrichtsmethoden II. Praxisband. Berlin, Cornelsen.

Pelikán, Jiří (2004): Základy empirického výzkumu pedagogických jevů. Praha, Univerzita Karlova v Praze/Karolinum.

Singule, František (1991): Americká pragmatická pedagogika: John Dewey a jeho následovníci. Praha, Státní pedagogické nakladatelství.

Sitná, Dagmar (2013): Metody aktivního vyučováni: spolupráce žákỉ ve skupinách. Praha, Portál.

Steiner, Rudolf (1993): Výchova ditète z hlediska duchovni védy. Praha, Baltazar.

Štástka, Tomáš (2019): Rezension (4. März): Divadlo pro všechny. Z Kytice jde na diváky doslova závrat. https:/www.idnes.cz/kultura/divadlo/kytice-recenze-narodni-divadloskutr.A190304_101501_divadlo_ts (25.07.2019).

Trier, Jost (1973): Das sprachliche Feld. Eine Auseinandersetzung. In: Trier, Jost / van der Lee, Anthony / Reichmann, Oskar (Hgg.): Aufsätze und Vorträge zur Wortfeldtheorie. Den Haag, De Gruyter Mouton.

Vávra, Zdeněk (2013): Integrace environmentálních témat do výuky cizich jazyků se zamérenim na jazyk německý. Disertační práce. Praha, Univerzita Karlova v Praze, Pedagogická fakulta.

Vondrová, Petra (2009): Výtvarné námèty pro čtvero ročních obdobi: pro dèti ve věku od 4 do 10 let. Praha, Portál.

Wittig, Rüdiger / Niekisch, Manfred (2014): Biodiversität: Grundlagen, Gefährdung, Schutz. Berlin Heidelberg, Springer-Verlag.

\author{
Zdeněk Vávra \\ Westböhmische Universität in Plzeň \\ Philosophische Fakultät \\ Lehrstuhl für Germanistik und Slawistik \\ Riegrova 11 \\ CZ-301 00 Plzeň \\ E-Mail: zvavra@kgs.zcu.cz
}

